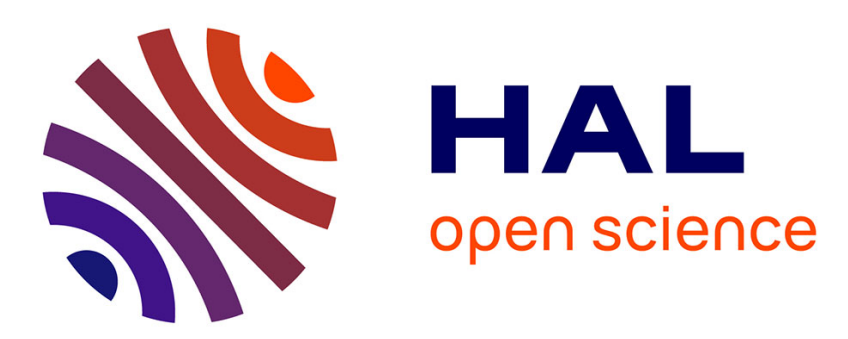

\title{
Constrained control of continuous-time minimum-phase systems. Global output reference tracking results
} Fouad Giri, Jean-Baptiste Gning, Fatima Zara Chaoui, E. Chater

\section{To cite this version:}

Fouad Giri, Jean-Baptiste Gning, Fatima Zara Chaoui, E. Chater. Constrained control of continuoustime minimum-phase systems. Global output reference tracking results. European Control Conference, ECC'2009, Aug 2009, Budapest, Hungary. pp.3142-3147. hal-01059999

\section{HAL Id: hal-01059999 https://hal.science/hal-01059999}

Submitted on 2 Sep 2014

HAL is a multi-disciplinary open access archive for the deposit and dissemination of scientific research documents, whether they are published or not. The documents may come from teaching and research institutions in France or abroad, or from public or private research centers.
L'archive ouverte pluridisciplinaire HAL, est destinée au dépôt et à la diffusion de documents scientifiques de niveau recherche, publiés ou non, émanant des établissements d'enseignement et de recherche français ou étrangers, des laboratoires publics ou privés. 


\title{
Constrained Control of Continuous-Time Minimum-Phase Systems Global Output Reference Tracking Results
}

\author{
F. GIRI, J.B. GNING, F.Z. CHAOUI", E. CHATER \\ GREYC Lab, University of Caen Basse-Normandie, Caen, France \\ * Corresponding author: chaouifatima@yahoo.fr
}

\begin{abstract}
Minimum phase systems are the only systems that allow (in disturbance-free unconstrained case) the achievement of perfect tracking in presence of arbitrary reference signals. The question is whether this still holds when the control input is subject to input saturation constraint. Surprisingly, most works on global output tracking in presence of input saturation focused on nonminimum phase systems. Then, perfect tracking is only achievable for constant references. In this paper, it is shown that more powerful tracking results are achievable for minimum phase systems. Specifically, perfect tracking is guaranteed for arbitrary type reference signals that satisfy a well defined strict compatibility condition. When, the reference signal is just compatible in the mean then the tracking quality depends on the reference variation rate. For periodic reference signals (not necessarily compatible with the constraint), all the closed-loop system signals are shown to be periodic with the same period.
\end{abstract}

Keywords: Minimum phase systems, input saturation constraint, output reference tracking, input-output stability, incremental stability.

\section{INTRODUCTION}

Tn this paper, the focus is made on global output reference tracking for stable linear systems in presence of input constraint. It is well known that such an issue is closely related to the system phase nature. In the unconstrained case, global tracking of arbitrary type reference signals is achievable only for minimum phase systems. The question is whether this still holds in the case of input saturation constraint. Surprisingly, early relevant results concerned nonminimum phase systems, e.g. ([2]-[3]). It was shown, using saturated versions of (adaptive) pole placement regulators, that perfect global tracking is only possible for constant reference signals that are strictly compatible with the input limitation. The problem of perfect global tracking of not necessarily constant references was dealt with in [9] considering minimum-phase stable systems controlled by saturated (adaptive) model reference regulator. It was shown that the tracking error converges globally to zero whatever the nature of the reference signal provided this is strictly compatible with the constraint. While such result constitutes a theoretical progress, its practical applicability is limited. In practical applications, the following tracking issues are important: (i) Does the regulator still show a tracking capability when facing reference signals that are only compatible in the mean (but not strictly)? (ii) How behaves the regulator in presence of reference signals with no compatibility feature? These issues are addressed in the present paper considering input-constrained minimum-phase systems controlled by saturated model reference regulators. In addition to perfect tracking of strictly compatible reference signals, it is shown that average tracking performances are ensured when the reference signal is just compatible in the mean. Then, the tracking error is proportional in the mean to the mean rate of the reference sequence. Furthermore, in the case of just periodic reference signals (not necessarily compatible), all closed loop signals are in steady-state periodic and oscillate with the same frequency as the reference.

The paper is organized as follows: Section 2 is devoted to formulating the control problem and designing the regulator; key technical lemmas are presented in Section 3 and used in Section 4 to establish the regulator tracking performances; a conclusion and reference list end the paper.

\section{Control Problem Formulation AND Regulator DESIGN}

We are considering continuous time SISO linear systems ${ }^{1}$ :

$$
A(s) y(t)=B(s) u(t) \quad(t \in I R)
$$

in presence of the input constraint:

$|u(t)| \leq u_{M}$

with:

$$
\begin{aligned}
& A(s)=s^{n}+a_{n-1} \cdot s^{n-1}+\ldots+a_{1} s+a_{0} \\
& B(s)=b_{n-1} \cdot s^{n-1}+\ldots+b_{1} s+b_{0}
\end{aligned}
$$

where $u(t)$ and $y(t)$ are the system input and output (respectively); $u_{M}$ denotes the maximal allowed control value; $s$ denotes as well the differential operator $\frac{d}{d t}$ or the Laplace variable; the integer $n$ and the real numbers $\left(a_{i}, b_{i}\right)$ are the system order and parameters, respectively. The polynomials $A(s)$ and $B(s)$ are Hurwitz i.e. the system is BIBO stable and minimum-phase. The stability assumption is required to make the system controllable in presence of the control limitation (1b). The minimum phase requirement is necessary because we seek perfect global tracking in presence of arbitrary piecewise continuous reference signals $y^{*}(t)$ that are compatible with the constraint (1b) (in a sense made precise later).

\footnotetext{
${ }^{1}$ Throughout the paper, IR denotes the set of real numbers.
} 
Let $R(s)$ and $S(s)$ the unique polynomials of the form:

$$
\begin{aligned}
& R(s)=s^{n-1}+r_{n-2} s^{n-2}+\ldots+r_{l} s+r_{0} \\
& S(s)=s_{n-1} s^{n-1}+s_{n-2} s^{n-2}+\ldots+s_{l} s+s_{0}
\end{aligned}
$$

such that:

$$
A(s) R(s)+S(s)=C(s)
$$

where $\mathrm{C}(s)$ is any Hurwitz polynomial of the form:

$$
C(s)=s^{2 n-1}+c_{2 n-2} s^{2 n-2}+\ldots+c_{1} s+c_{0}
$$

The saturated model-reference regulator we are proposing consists in generating an auxiliary control signal $v(t)$ according to the following control law:

$$
A(s) B(s) v(t)=[\Lambda(s) B(s)-B(s) R(s)] u(t)-S(s) y(t)+C(s) y^{*}(t) \quad(5 \mathrm{a})
$$

where $\Lambda(s)$ is any Hurwitz polynomial of the form:

$$
\Lambda(\mathrm{s})=s^{\mathrm{n}-1}+\lambda_{\mathrm{n}-2} s^{n-2}+\ldots+\lambda_{1} \mathrm{~s}+\lambda_{0}
$$

and letting the control action $u(t)$ be:

$$
u(t)=\operatorname{sat}(v(t)) \stackrel{\text { def }}{=} \operatorname{sign}(v(t)) \cdot \min \left\{u_{M},|v(t)|\right\}
$$

\section{Remarks 2.1.}

1) The saturated regulator $(5 a-b)$ coincides with the standard linear model-reference regulator:

$$
B(s) R(s) u(t)+S(s) y(\mathrm{t})=\mathrm{C}(s) y^{*}(t)
$$

whenever the control signal stops saturating for a long time. Then, one gets $\mathrm{C}(s) e(t)=0$ where $e(t) \stackrel{d e f}{=} y(t)-y^{*}(t)$ is the tracking error. The error $e(t)$ then vanishes exponentially fast because $C(s)$ is Hurwitz. This determines the regulation dynamics of the closed-loop system.

2) The reference signal $y^{*}(t)$ may be the output of a model reference system, e.g. $y^{*}(t)=u_{m}(t) / A_{m}(s)$ where $u_{m}(t)$ denotes the ideal output reference and $A_{m}(s)$ is a Hurwitz polynomial such that $A_{m}(0)=1$. The transfer function $1 / A_{m}(s)$ is then referred to tracking dynamics of the closed-loop system. The point is that the regulation dynamics (defined by $C(s)$ ) and the tracking dynamics are presently independently chosen.

3) The above regulator design is quite different from the state-space design proposed in [8]. In the latter the tracking dynamics are identical to the regulation dynamics $\left(A_{m}(s)=C(s)\right)$.

\section{Technical ToOls And Preliminary Results}

In this section, we recall a number of technical tools relevant to the theory of input-output stability, e.g. [8]. In particular, Lemma 3.1 will play a central role in the analysis of the next section.

\section{A. Preliminary Notions}

Let $\Omega$ denotes the linear space of all causal real signals (i.e. $s: I R \rightarrow I R)$. The $L_{p}$ norm of $x \in \Omega$ is denoted $x_{p}$ $(1 \leq p \leq \infty)$. For any integer $T>0, s_{T}$ designates the truncated signal i.e. $x_{T}(t)=x(t)$ for $0 \leq t \leq T$ and $x_{T}(t)=0$ for $t>T$.

Definition 3.1 (Sectoricity). A dynamic nonlinear map $\phi$ : $I R \times I R \rightarrow I R$ belongs to the sector $[a, b]$ (with $a<b)$, if: $a z^{2} \leq z \phi(t, z) \leq b z^{2}, \quad$ for all $(t, z) \in I R^{2}$

The set of such functions is denoted $S[a, b]$

Definition 3.2 (stability). Let $H: \Omega \rightarrow \Omega$ be any dynamic (non) linear operator.

1) $H$ is $L_{p}$-stable (for some $1 \leq p \leq \infty$ ) if there exists a real $\gamma$ such that: $\|H u\|_{p} \leq \gamma\|u\|_{p}$, for all $u \in L_{p}$. The smallest $\gamma$ is called $L_{p}$-gain of $H$ and is denoted $\gamma_{p}(H)$ or simply $\gamma_{p}$.

2) $H$ is $L_{p}$-incrementally stable if it is $L_{p}$-stable and there is a real $\tilde{\gamma}$ such that $\left\|H u_{1}-H u_{2}\right\|_{p} \leq \tilde{\gamma}\left\|u_{1}-u_{2}\right\|_{p}$, whatever $u_{1}, u_{2} \in L_{p}$. The smallest $\tilde{\gamma}$ is called incremental $L_{p}$ gain and is denoted $\tilde{\gamma}_{p}(H)$ or simply $\tilde{\gamma}_{p}$

The next definitions make precise the sense of (reference signal) compatibility and signal smallness in the mean (e.g. [4]). In these definitions (and throughout), $m(T)$ denotes the measure of subset $T \subset I R$. Specifically:

$m\left(\left[t_{0} t_{1}\right]\right)=t_{1}-t_{0} \quad$ (for all real numbers $\left.t_{0} \leq t_{1}\right)$ and $m\left(T_{1} \cup T_{2}\right)=m\left(T_{1}\right)+m\left(T_{2}\right)$ for any subsets $T_{1}, T_{2} \subset I R$ such that $T_{1} \cap T_{2}$ is empty.

Definition 3.3. Let $\alpha$ be any real number and $x \in \Omega$ be any real signal.

1) $x$ is said $\alpha$-small in the mean (briefly $\alpha-S M$ ), if: $\limsup _{m(T) \rightarrow \infty} \frac{1}{m(T)} \int_{T} x(t) d t \leq \alpha$

2) Given $\alpha$, the set of $\alpha$-SM signals is denoted $S M(\alpha)$

In the above definition and throughout, the notations $x \in S M(\alpha)$ and $x(t) \in S M(\alpha)$ are indifferently used.

Definition 3.4. Consider the system (1a). Let $y^{*} \in \Omega$ be any piecewise-continuous bounded signal and $u^{*} \in \Omega$ be any signal obtained, from $y^{*}$, solving the following differential equation:

$B(s) u^{*}(t)=A(s) y^{*}(t)$,

with arbitrary finite initial values $\left(d^{i} u^{*} / d t^{i}\right)(0)$ $(i=1 \ldots n-2)$.

1) $y^{*}$ is compatible in the mean with the constraint (1b) if $u^{*}(t) \quad$ is bounded and $\left|u^{*}(t)\right| \in S M\left(u_{M}\right)$, i.e. $\limsup _{m(T) \rightarrow \infty} \frac{1}{\mathrm{~m}(\mathrm{~T})} \int_{T}\left|\mathrm{u}^{*}(\mathrm{t})\right| d t \leq u_{M}$.

2) $y^{*}$ is strictly compatible with the constraint if there is a real $0<\delta<1$ such that $\left|u^{*}(t)\right| \leq u_{M}(1-\delta)$ (for all $\left.t\right)$ 
Remark 3.1. The signal $u^{*}(t)$ generated by (8) is referred to control signal induced by the reference $y^{*}(t)$. A reference that is compatible in the mean allows the induced control to press on the constraint or even to violate it from time to time. Unlikely, the control signal induced by a strictly compatible reference does never violate the constraint nor it presses on it. The type of reference signal compatibility determines the achievable tracking performances (Theorems 4.1 and 4.2)

\section{B. Technical Lemmas.}

Lemma 3.1 (Properties of the saturation function).

The function sat(.) has the following properties:

1) $\operatorname{sat}(.) \in S[0,1]$.

2) $|v|-|\operatorname{sat}(v)|=|v-\operatorname{sat}(v)|, \quad($ for all $v \in I R)$

3) $|v-\operatorname{sat}(v)| \neq 0 \Rightarrow \operatorname{sat}(v)=u_{M} \operatorname{sign}(v),($ for all $v \in I R)$

4) For all $v_{1}, v_{2} \in I R$, there exist $\xi \in[0,1]$ such that:

$\operatorname{sat}\left(v_{1}\right)-\operatorname{sat}\left(v_{2}\right)=\xi\left(v_{1}-v_{2}\right)$

Proof. See e.g. [2], [3].

Lemma 3.2. Let $x, x_{1}, x_{2} \in \Omega$ be any real signals and $\mu, \mu_{1}, \mu_{2}, k_{1}, k_{2}$ be any real numbers.

1) If $x_{1}^{2} \in S M\left(\mu_{1}\right)$ and $x_{2}^{2} \in S M\left(\mu_{2}\right)$ then:

$\left(k_{1} x_{1}+k_{2} x_{2}\right)^{2} \in S M\left(2 k_{1}^{2} \mu_{1}+2 k_{2}^{2} \mu_{2}\right)$

2) If $x^{2} \in S M(\mu)$ then $|x| \in S M(\sqrt{\mu})$

3)Let $H$ be any $L_{p}$-stable operator $(p=1,2)$. If $x^{p} \in S M(\mu)$ then $(H x)^{p} \in S M\left(\left(\gamma_{p}(H)\right)^{p} \mu\right)$

Proof. See detailed version of this paper

Lemma 3.3. Consider the feedback system of Fig 3.1 involving a proper Hurwitz transfer function $G(s)$ in closed-loop with the nonlinear map $\psi: I R \rightarrow I R$, $z \rightarrow z-\operatorname{sat}(z)$. Then, one has the following properties:

1) The feedback is $L_{\infty}$-stable if $\gamma_{a}<2$ with:

$$
\gamma_{a}=\gamma_{\infty}\left(\frac{G(s)}{1+G(s) / 2}\right)
$$

Then one has:

$$
\left\|e_{2}\right\|_{\infty} \leq \frac{\gamma_{a}}{1-\frac{\gamma_{a}}{2}}\left\|u_{1}\right\|_{\infty}+\frac{1+\frac{\gamma_{a}}{2}}{1-\frac{\gamma_{a}}{2}}\left\|u_{2}\right\|_{\infty}
$$

2) The feedback is $L_{2}$-stable if:

$$
\inf _{0 \leq \omega<\infty} \operatorname{Re}(G(j \omega))>-1
$$

3) Under condition (12), the nonlinear map $w \rightarrow e_{2}$ is $L_{2}$ incrementally stable, with $w=u_{2}+G(s) u_{1}$

Proof. See e.g. [9]

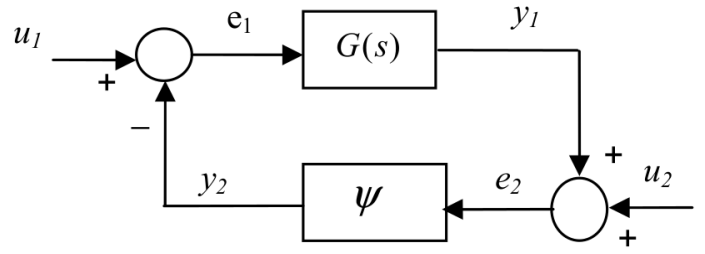

Fig 1. Feedback system in Lemma 3.1

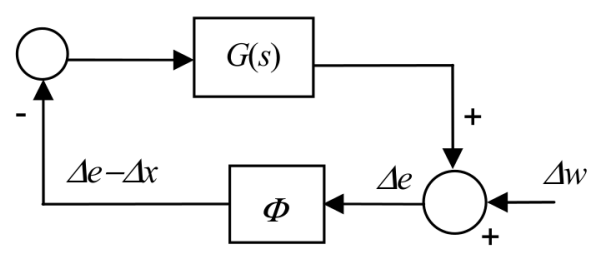

Fig 2. Feedback system defined by equ. (15)

\section{ANAlysis OF the SATURATED REgulator PERFORMANCES}

In this section, the tracking capability of the saturated regulator defined by (5a-b) is analyzed using the technical tools presented in Section 3. Quite interesting results are established for different classes of reference signals. First, we investigate the properties of operator $y^{*} \rightarrow v$.

Proposition 4.1. Consider the constrained system (1a-b) in closed-loop with the saturated regulator $(5 a-b)$. Then, the nonlinear dynamic map $y^{*} \rightarrow v$ has the feedback structure of Fig 1 with:

$u_{1}=0, e_{2}=v, y_{2}=v-u$

$u_{2}(t)=u^{*}(t)+\varepsilon(t)=\frac{A(s)}{B(s)} y^{*}+\varepsilon(t)$

$G(s)=\frac{\Lambda(s) A(s)-C(s)}{C(s)}$

$\psi: I R \rightarrow I R, z \rightarrow z-\operatorname{sat}(z)$

where $u^{*}(t)$ is as in Definition 3.4 and $\varepsilon(t)$ is an exponentially vanishing term

Proof. See [10]

Proposition 4.2. Consider the constrained system (1a-b) in closed-loop with the saturated regulator $(5 a-b)$. Then, one has the following properties:

1) If the reference signal $y^{*}$ is bounded, all signals of the closed-system are bounded.

2) If the reference signal $y^{*}$ is periodic, all signals of the closed-loop system are periodic (in steady state) with the same period as $y^{*}$

\section{Proof. See [10]}

Remark 4.1. Part 2 of the above proposition, shows that the behavior of closed-loop system is almost linear as the response to any periodic input is (in steady state) periodic 
with the same period. This is a quite interesting feature of the present paper compared to recent works, e.g. [9]. The key step to achieve such result is the proof of incremental stability for the operator $H: u^{*}(t)+\varepsilon(t) \rightarrow v(t)$. Finally, notice that Propositions 4.1 and 4.2 do not require the reference signal to be compatible with the constraint (Definition 3.4)

Theorem 4.1 (Tracking strictly compatible reference signals). Consider the constrained system (1a-b) in closedloop with the saturated regulator $(5 a-b)$. Let the reference signal $y^{*}$ be strictly compatible with the constraint (1b) and let $C(s)$ be chosen so that the following two conditions are satisfied:

$\gamma_{b}<1$

$\frac{1+\gamma_{b}}{1-\gamma_{b}}<1+\delta$

with

$\gamma_{b} \stackrel{\text { def }}{=} \gamma_{\infty}\left(\frac{A(s) \Lambda(s)-C(s)}{A(s) \Lambda(s)+C(s)}\right)$

where $\delta$ is as in Definition 3.4 (Part 2). Then, there exists an integer $T \in I R$, such that for all $t \geq T$ :

1) $|v(t)|<u_{M}$ and, consequently, $u(t)=v(t)$

2) $C(s)\left(y(\mathrm{t})-y^{*}(t)\right)=0$ and, so, $\lim _{t \rightarrow \infty}\left(y(\mathrm{t})-y^{*}(t)\right)=0$

Proof. See e.g. [10]

\section{Remark 4.2.}

Note that conditions (33a-b) can be fulfilled choosing $C(s)$ and $\Lambda(s)$ such that $C(s)$ is sufficiently close to $A(s) \Lambda(s)$. Indeed, it is seen from (15) that

$$
\lim _{C(s) \rightarrow A(s) \Lambda(s)} \gamma_{b}=0
$$

Theorem 4.2 (Tracking of mean compatible reference signals). Consider the constrained system (1a-b) in closedloop with the saturated regulator (5a-b). Suppose that:

$\inf _{0 \leq \omega<\infty} \operatorname{Re} \frac{A(j \omega) \Lambda(j \omega)}{C(j \omega)}>0$

Then, if the signal $y^{*}$ is compatible in the mean with the constraint, one has:

$$
\begin{aligned}
& \text { 1) }(\dot{v})^{2},(\dot{u})^{2} \in S M\left(K^{*} \mu\right) \text {; } \\
& \text { 2) }|v-u| \in S M\left(K^{*} \sqrt{\mu}\right) \\
& \text { 3) }\left|y-y^{*}\right| \in S M\left(K^{*} \sqrt{\mu}\right)
\end{aligned}
$$

where $K^{*}>0$ is a generic constant independent of $\mu$

Proof. Part 1. Operating $\frac{d}{d t}$ on both sides of (22) gives:

$$
\dot{v}(t)=-\frac{A(s) \Lambda(s)-C(s)}{C(s)}(\dot{v}(t)-\dot{u}(t))+\frac{A(s)}{B(s)} \dot{y}^{*}(t)+\dot{\varepsilon}(t)
$$

where $\dot{\varepsilon}(t)$ is an exponentially vanishing term (because so is $\varepsilon(t))$. On the other hand, let $\phi$ denotes the nonlinear dynamic map:

$$
\phi: I R \rightarrow I R ; \quad \dot{v}(t) \rightarrow \dot{v}(t)-\dot{u}(t)
$$

Then, the system (17)-(18) fits the feedback of Fig 1 with:

$u_{1}=0, e_{2}=\dot{v}, y_{2}=\dot{v}-\dot{u}$

$u_{2}(t)=\frac{A(s)}{B(s)} \dot{y}^{*}(t)+\dot{\varepsilon}(t)$

$G(s)=\frac{A(s) \Lambda(s)-C(s)}{C(s)}$

Notice that $(\dot{\varepsilon})^{2} \in S M(0)$, because $\dot{\varepsilon}(t)$ is exponentially vanishing. Then, applying Lemma 3.2 (Part 1), it follows from (37) and (20) that:

$\left(u_{2}\right)^{2} \in S M\left(K_{1} \mu\right)$

Now, let us check that $\phi \in S[0,1]$. Indeed, one has from equation (5b):

$\dot{u}(t)=\frac{d u(t)}{d t}=\frac{d u}{d v} \frac{d v}{d t}=\alpha(v) \cdot \dot{v}(t)$

with

$$
\alpha(v)=\frac{d u}{d v}=\left\{\begin{array}{c}
0 \text { if } v(t)>u_{M} \\
1 \text { if }|v(t)| \leq u_{M} \\
0 \text { if } v(t)<-u_{M}
\end{array}\right.
$$

This shows that:

$0 \leq \alpha(v) \leq 1$

Using (24), it follows from (23) that:

$\dot{\mathrm{v}}(\mathrm{t})(\dot{\mathrm{v}}(\mathrm{t})-\dot{\mathrm{u}}(\mathrm{t}))=(\dot{\mathrm{v}}(\mathrm{t}))^{2}(1-\alpha(v)) \leq(\dot{\mathrm{v}}(\mathrm{t}))^{2}$

Hence, $\phi \in S[0,1]$. Applying Lemma 3.3 (Part 2) to the feedback of Fig 1 with the definitions (19)-(21), it follows that $L_{2}$-stability would be guaranteed for that feedback, if:

$$
\inf _{0 \leq \omega<\infty} \operatorname{Re}(G(j \omega))>-1
$$

which, in view of (13a), becomes:

$\inf _{0 \leq \omega<\infty} \operatorname{Re}\left(\frac{A(j \omega) \Lambda(j \omega)}{C(j \omega)}-1\right)>-1$

But, this actually holds, due to (16). That is, the feedback of Fig 1 (with notations (19)-(21)) is actually $L_{2}$-stable. As a consequence of $L_{2}$-stability and Lemma 3.2 (Part 2), it follows from (22) and (19)-(20) that there exists a constant $K_{2}>0$ such that $\left(e_{2}\right)^{2} \in S M\left(K_{2} \mu\right)$ which in turn gives using the fact that $\dot{v}=e_{2}$ (see (20)):

$(\dot{\mathrm{v}})^{2} \in S M\left(K_{2} \mu\right)$

Using (23-25), one gets from (28):

$(\dot{u})^{2} \in S M\left(K_{2} \mu\right)$

Part 1 of the proposition follows from (28)-(29) with any $K^{*} \geq K_{2}$.

Proof of Part 2. Using (8), equation (22) can be rewritten:

$$
\frac{\Lambda(s) A(s)}{C(s)}(v(t)-u(t))=u^{*}(t)-u(t)+\varepsilon(t)
$$

This immediately gives:

$$
\begin{array}{r}
\frac{A(0) \Lambda(0)}{C(0)}(v(\mathrm{t})-\mathrm{u}(\mathrm{t}))=\left(\frac{A(0) \Lambda(0)}{C(0)}-\frac{A(s) \Lambda(s)}{C(s)}\right)(v(\mathrm{t})-\mathrm{u}(\mathrm{t})) \\
+u^{*}(t)-u(t)+\mathcal{E}(t)
\end{array}
$$


$=\left(\frac{A(0) \Lambda(0) C(s)-C(0) A(s) \Lambda(s)}{C(0) C(s)}\right)(v(\mathrm{t})-\mathrm{u}(\mathrm{t}))$

$$
+u^{*}(t)-u(t)+\varepsilon(t)
$$

It is readily seen that the polynomial $[A(0) \Lambda(0) C(s)-C(0) A(s) \Lambda(s)]$ has a zero at $s=0$. Then, there is a unique polynomial $Q(s)$ such that:

$A(0) \Lambda(0) C(s)-C(0) A(s) \Lambda(s)=s Q(s)$. Then, it follows from (31):

$$
\begin{aligned}
\frac{A(0) \Lambda(0)}{C(0)}(v(\mathrm{t})-\mathrm{u}(\mathrm{t})) & =\frac{Q(s)}{C(0) C(s)}(\dot{\mathrm{v}}(\mathrm{t})-\dot{u}(\mathrm{t}))+u^{*}(t)-u(t)+\varepsilon(t) \\
& =u^{*}(t)-u(t)+\varepsilon_{1}(t)
\end{aligned}
$$

with

$\varepsilon_{1}(t) \stackrel{\text { def }}{=} \frac{Q(s)}{C(0) C(s)}(\dot{v}(t)-\dot{u}(t))+\varepsilon(t)$

Notice that the transfer function $\frac{Q(s)}{C(0) C(s)}$ is $L_{2}$-stable because $C(s)$ is Hurwitz. Then, applying Lemma 3.2 (Part $2)$, it follows from (28)-(29) that:

$$
\left(\varepsilon_{1}(t)\right)^{2} \in S M\left(K_{3} \mu\right)
$$

where we have also used the fact that $\varepsilon(t)$ is exponentially vanishing. Now, let show that if $v(t)-u(t) \neq 0$, then:

$$
\frac{A(0) \Lambda(0)}{C(0)}|(v(\mathrm{t})-\mathrm{u}(\mathrm{t}))| \leq\left|\varepsilon_{1}(t)+u^{*}(t)\right|-u_{M}
$$

First, notice that $A(0)>0, \Lambda(0)>0, C(0)>0$. This readily follows applying the Routh's criterion to the Hurwitz polynomials $A(s), \Lambda(s), C(s)$. To prove (35), let us examine the three cases: $v(t)<-u_{M},|v(t)| \leq u_{M}, v(t)>u_{M}$.

Case 1: $\quad v(t)<-u_{M} . \quad$ Then, $\quad u(t)=-u_{M} \quad$ and $v(t)-u(t)=v(t)+u_{M}<0$. This, together with (32), gives:

$0>\frac{A(0) \Lambda(0)}{C(0)}(v(t)-u(t)) \geq u^{*}(t)+\varepsilon_{1}(t)+u_{M}$

or, equivalently:

$0<\frac{A(0) \Lambda(0)}{C(0)}(-v(\mathrm{t})+\mathrm{u}(\mathrm{t})) \leq-\left(u^{*}(t)+\varepsilon_{1}(t)\right)-u_{M}$

It is readily seen from $(36)$ that:

$(-v(t)+u(t))>0$ and $-\left(u^{*}(t)+\varepsilon_{1}(t)\right)>0$

Then, (36) implies (35).

Case 2: $\quad v(t)>u_{M} . \quad$ Then, $\quad u(t)=u_{M} \quad$ and $v(t)-u(t)=v(t)-u_{M}>0$. This, together with (33), gives:

$0 \leq \frac{A(0) \Lambda(0)}{C(0)}(v(t)-u(t)) \leq u^{*}(t)+\varepsilon_{1}(t)-u_{M}$

It readily follows from $(37)$ that:

$(v(t)-u(t))>0$ and $u^{*}(t)+\varepsilon_{1}(t)>0$

This, together with (37), implies (35). Hence, the statement (35) does hold.

Now, let $T_{0} \subset I R$ denotes the subset including all time instants at which $v(t)-u(t)=0$ and $T_{1}=I R-T_{0}$. It readily follows from these definitions that, $T_{0} \cup T_{1}=I R, T_{0} \cap T_{1}$ is empty and, for all $T \subset I R$ :

$$
\begin{aligned}
\frac{1}{m(T)} \int_{T} \mid(v(\mathrm{t})-\mathrm{u}(\mathrm{t})) & \left|d t=\frac{1}{m(T)} \int_{T \cap T_{1}}\right|(v(\mathrm{t})-\mathrm{u}(\mathrm{t})) \mid d t \\
& =\frac{m\left(T \cap T_{1}\right)}{m(T)} \frac{1}{m\left(T \cap T_{1}\right)} \int_{T \cap T_{1}}|(v(t)-u(t))| d t \\
& \leq \frac{1}{m\left(T \cap T_{1}\right)} \int_{T \cap T_{1}}|(v(t)-u(t))| d t
\end{aligned}
$$

because $m\left(T \cap T_{1}\right) / m(T) \leq 1$. Using (38), it follows from (35) that:

$\frac{A(0) \Lambda(0)}{C(0)} \frac{1}{m(T)} \int_{T}|(v(\mathrm{t})-\mathrm{u}(\mathrm{t}))| d t \leq \frac{1}{m(T)} \int_{T \cap T_{1}}\left(\left|\varepsilon_{1}(t)+u^{*}(t)\right|-u_{M}\right) d t$ $\leq \frac{1}{m(T)} \int_{T \cap T_{1}}\left(\left|\varepsilon_{1}(t)\right|\right) d t+\frac{1}{m(T)} \int_{T \cap T_{1}}\left(\left|u^{*}(t)\right|-u_{M}\right) d t$ (39)

At this point, three cases have to be distinguished.

a) $m\left(T_{1}\right)<\infty$. Then, the integrals on the right side of (39) are finite because all integrated signals are bounded and $m\left(T \cap T_{1}\right)<\infty$, whatever $T \subset I R$. Consequently,

$\limsup _{m(T) \rightarrow \infty} \frac{1}{m(T)} \int_{T}|(v(t)-u(t))| d t=0$

b) $m\left(T_{1}\right)=\infty$ and $\limsup _{m(T) \rightarrow \infty} m\left(T \cap T_{1}\right)<\infty$. Then, one has:

$\frac{1}{m(T)} \int_{T \cap T_{1}}\left|\varepsilon_{1}(t)\right| d t=\frac{m\left(T \cap T_{1}\right)}{m(T)} \frac{1}{m\left(T \cap T_{1}\right)} \int_{T \cap T_{1}}\left|\varepsilon_{1}(t)\right| d t$

which implies:

$\limsup _{m(T) \rightarrow \infty} \frac{1}{m(T)} \int_{T \cap T_{1}}\left|\varepsilon_{1}(t)\right| d t=0$

because all integrated signals on the right side of (41) are bounded and $\limsup _{m(T) \rightarrow \infty} \frac{m\left(T \cap T_{1}\right)}{m(T)}=0$. Similarly, one can show that:

$\limsup _{m(T) \rightarrow \infty} \frac{1}{m(T)} \int_{T \cap T_{1}}\left(\left|u^{*}(t)\right|-u_{M}\right) d t=0$

Combining (42a-b), one gets from (40) that:

$\limsup _{m(T) \rightarrow \infty} \frac{1}{m(T)} \int_{T}|(v(\mathrm{t})-\mathrm{u}(\mathrm{t}))| d t=0$

c) $m\left(T_{1}\right)=\infty \quad$ and $\quad \limsup m\left(T \cap T_{1}\right)=\infty$. Then, as $m\left(T \cap T_{1}\right) / m(T) \leq 1$, one gets from (41) that:

$\limsup _{m(T) \rightarrow \infty} \frac{1}{m(T)} \int_{T \cap T_{1}}\left|\mathcal{E}_{1}(t)\right| d t \leq \limsup _{m(T \cap T) \rightarrow \infty} \frac{1}{m\left(T \cap T_{1}\right)} \int_{T \cap T_{1}}\left|\mathcal{E}_{1}(t)\right| d t$

$$
\leq \sqrt{K_{3} \mu}
$$

The last inequality follows from (34), using Lemma 3.2 (Part 2). Similarly, one has:

$$
\begin{aligned}
\frac{1}{m(T)} \int_{T \cap T_{1}}\left(\left|u^{*}(t)\right|-u_{M}\right) d t \\
\quad=\frac{m\left(T \cap T_{1}\right)}{m(T)} \frac{1}{m\left(T \cap T_{1}\right)} \int_{T \cap T_{1}}\left(\left|u^{*}(t)\right|\right) d t-u_{M} \\
\leq \frac{1}{m\left(T \cap T_{1}\right)} \int_{T \cap T_{1}}\left(\left|u^{*}(t)\right|\right) d t-u_{M}
\end{aligned}
$$

Therefore, 


$$
\begin{aligned}
& \limsup _{m(T) \rightarrow \infty} \frac{1}{m(T)} \int_{T \cap T_{1}}\left(\left|u^{*}(t)\right|-u_{M}\right) d t \\
& \quad \leq \limsup _{m\left(T \cap T_{1}\right) \rightarrow \infty} \frac{1}{m\left(T \cap T_{1}\right)} \int_{T \cap T_{1}}\left(\left|u^{*}(t)\right|\right) d t-u_{M} \leq 0
\end{aligned}
$$

The last inequality follows from the fact that the reference signal $y^{*}$ is compatible in the mean with the constraint (Definition 3.4, Part 1). Using (64a-b), it follows from (38) that:

$$
\limsup _{m(T) \rightarrow \infty} \frac{1}{m(T)} \int_{T}|(v(t)-u(t))| d t \leq \frac{C(0)}{A(0) \Lambda(0)} \sqrt{K_{3} \mu}
$$

Combining the results (40), (63) and (65), it follows that $|(v(t)-u(t))| \in S M\left(K_{4} \sqrt{\mu}\right)$ with $K_{4}=\frac{C(0)}{A(0) \Lambda(0)} \sqrt{K_{3}}$. This establishes Part 2 of Theorem 4.2.

Proof of Part 3. The control law (5a) can be rewritten: $B(s) R(s) u(t)+S(s) y(t)=C(s) y^{*}(t)+\Lambda(s) B(s)(u(t)-v(t))(46)$ In view of $(1 \mathrm{a}), B(s) u(\mathrm{t})$ can be substituted to $A(s) y(\mathrm{t})$ in the left side of (66). Doing so, there exists $t_{0}>0$ such that : for all $t \geq t_{0}$ :

$R(s) A(s) y(t)+S(s) y(t)=C(s) y^{*}(t)+\Lambda(s) B(s)(u(t)-v(t))$

Using (4a), this yields:

$C(s) y(t)=C(s) y^{*}(t)+\Lambda(s) B(s)(u(t)-v(t))$

or, equivalently:

$y(t)-y^{*}(t)=\frac{\Lambda(s) B(s)}{C(s)}(u(t)-v(t))$

$\Lambda(s) B(s) / C(s)$ is $L_{1}$-stable (because $C(s)$ is Hurwitz). As $|(v(t)-u(t))| \in S M\left(K_{4} \sqrt{\mu}\right)$, it follows applying Lemma 3.2 (Part 3) to (67), that $\left|y-y^{*}\right| \in S M\left(K_{5} \sqrt{\mu}\right)$ with $K_{5}=K_{4} \gamma_{1}(\Lambda(s) B(s) / C(s))$. This establishes Part 3 and completes the proof of Theorem 4.2

\section{Remark 4.3.}

1) The SPR condition (16) defines (just as did condition (14a-b)) a neighborhood of the controlled system poles in which one should assign the closed-loop system regulation poles. Such requirement guarantees the average tracking performances of Theorem 4.2. That is, the mean size of the control error $(v-u)$ and the tracking error $\left(y-y^{*}\right)$ are proportional to the mean rate of the reference signal. The smaller the latter the better is the average tracking quality.

2) Compared with Theorem 4.1, Theorem 4.2 does not ensure perfect tracking (convergence of tracking error to zero) but it involves a much wider class of reference signals and the control signal is allowed to press and even violate the constraint infinitely often.

3) The concept of compatibility in the mean (Definition 3.4) is an original feature of the present paper. Indeed, the existing tracking results, for input constrained stable linear systems, are limited to strictly compatible reference signals, e.g. ([9], [3])

\section{Simulations}

The simulation results are omitted due to space limitations. They will be presented in the conference.

\section{CONCLUSION}

We have considered the problem of controlling constrained continuous-time minimum-phase linear systems. The original saturated regulator $(5 \mathrm{a}-\mathrm{b})$ provides powerful tracking properties. Specifically, in presence of reference signals that are strictly compatible with the constraint (Definition 4.4), the regulator ensures perfect output reference tracking (Theorem 4.1). In presence of reference signals that are compatible in the mean (Definition 3.4), the tracking quality depends on the reference variation: the slower the reference signal, the better the tracking quality (Theorem 4.2). Finally, in the case of just periodic reference signals (not necessarily compatible with the constraint), all signals of the closed-loop system are in turn periodic with the same period as the driving reference (Proposition 4.2). It is the first time that such a high level of tracking performances is achieved in present of input constraint.

\section{REFERENCES}

[1] Blanchini F., (1999). Set invariance in control - A Survey. Automatica, vol 35, pp. 1747-1767.

[2] Chaoui F.Z, F. Giri, M. M'Saad, (2001). Adaptive control of inputconstrained type-1 plants: stabilization and tracking. Automatica, Vol. 37, pp. 197-203.

[3] Chaoui F.Z., F. Giri, L. Dugard, J.M. Dion, M. M'Saad, (1998). 'Adaptive tracking with saturating input and controller integral action', IEEE-TAC, Vol.43, pp. 1638-1643.

[4] Giri, F., M. M'Saad, L. Dugard and J.M. Dion, (1988). Robust pole placement indirect adaptive control. Int. J. Adaptive Contr. Signal Processing, vol.2, pp.33-47.

[5] Glattfelder A.H. and W. Schaufelberger (2003). Control systems with input and output constraints. Springer, Berlin, (ISBN-10: 1852333871).

[6] Khalil H., Nonlinear Systems. Prentice Hall, Inc., N.J., USA., 2003.

[7] Sussmann H. J., E.D. Sontag, and Y. Yang, (1994). A general result on the stabilization of linear systems using bounded controls. IEEE-TAC, vol.AC-39, $\mathrm{n}^{\circ} 12$, pp.2411-2425.

[8] Vidyasagar M., (2002). Nonlinear Systems Analysis. 2d edition, SIAM, PA, USA. ISBN: 0-89871-526-1

[9] Zhong Y.S. (2005). Globally stable adaptive system design for minimum phase SISO plants with input saturation. Automatica, vol. 41, pp. 1539-1547.

[10] The detailed version of this paper 